\title{
Para Além da Atenção Básica: reorganização do SUS por meio da interseção do setor político com o econômico
}

\author{
Beyond Primary Care: reorganization of SUS through the \\ intersection of the political and economic sectors
}

\author{
Leila Bernardo Donato Göttems \\ Doutoranda em Administração da Universidade de Brasília. \\ Professora do Curso de Enfermagem da Universidade Católica \\ de Brasília. \\ Endereço: SHCES Q. 1109, BI. D, Apt. I0I, Cruzeiro Novo, CEP 70658 \\ 190, Brasília, DF, Brasil. \\ E-mail: leiladळucb.br; leila.gottemsळuol.com.br \\ Maria Raquel Gomes Maia Pires \\ Doutora em Política Social. Professora Adjunta do Departamento de \\ Enfermagem Aplicada da Escola de Enfermagem da Universidade \\ Federal de Minas Gerais. \\ Endereço: Escola de Enfermagem/Campus Saúde/UFMG, Av. Alfre- \\ do Balena, 190, sala 514, Bairro Santa Efigênia, CEP 31130100 , Belo \\ Horizonte, MG, Brasil. \\ E-mail: maiapœuol.com.br; raquelgmpœenf.ufmg.br
}

\section{Resumo}

Questionam-se os limites e as possibilidades para reestruturar os serviços de saúde do Sistema Único de Saúde (SUS) a partir da atenção básica à saúde. Parte-se da hipótese de que a atenção básica à saúde, ação integrada de promoção, prevenção e recuperação da saúde da população, embora seja espaço político para produção de saberes e tecnologias partilhadas de poder, tem pouca influência no reordenamento do mercado em saúde no Brasil, comprometendo seu potencial para inversão do modelo de atenção. Este artigo tem como objetivos contextualizar a atenção básica na gestão da assistência à saúde no SUS; refletir teoricamente sobre a reorganização dos serviços a partir da atenção primária à saúde (APS), considerando as dimensões econômicas das políticas sociais. A pesquisa teórica e documental teve abordagem qualitativa. Fez-se análise crítica de documentos oficiais, nacionais e internacionais. Conclui-se pela necessidade de uma atenção básica melhor articulada à média e à alta complexidades, capaz de interferir na lógica da oferta a partir da demanda e reduzir iniquidades.

Palavras-chave: Atenção primária à saúde; Sistema Único de Saúde; Serviços de saúde. 


\section{Abstract}

The limits and possibilities concerning the restructuring of the health services of Sistema Único de Saúde (SUS - National Health System) are discussed based on primary care. The hypothesis is that primary care, which means integrated action of promotion, prevention and recuperation of the population's health, despite the fact that it is a political space for the production of knowledge and of power-sharing technology, it has little influence on the reorganization of the health market in Brazil, which negatively affects its potential concerning the inversion of the primary care model. This paper aims to contextualize primary care in the health assistance management of SUS, and to think theoretically about the reorganization of the SUS services starting from primary health care, and taking into account the economic dimensions of the social policies. The theoretical and documental research had a qualitative approach. Critical analysis of official documents, both national and international, was performed. The conclusion is for the need of a primary care that is better articulated with the medium and high complexities, able to interfere in the logic of the offer based on the demand and to reduce inequities.

Keywords: Primary Health Care; Brazil's National Health System; Health Services.

\section{Introdução}

Apesar dos avanços na formulação e descentralização das políticas de saúde, fruto do movimento da Reforma Sanitária, a inversão do paradigma assistencial permanece como utopia concretizável para o SUS. A referida mudança consistiria em transcender a abordagem curativa, hospitalocêntrica, fragmentada em especialidades, fundada em processos de trabalhos rigidamente divididos e na hegemonia do médico sobre a equipe de saúde (Pires, 1989; Merhy, 1997). Em seu lugar, propõem-se abordagens interdisciplinares, com resgate da integralidade da atenção, centrada na saúde, na comunidade, no fortalecimento das redes solidárias, na coparticipação social e na pessoa como sujeito do processo de saúde-doença, seja em nível individual ou coletivo. Esse tipo de resposta aos problemas de saúde transcende o enfoque biológico e se respalda na vigilância à saúde, concepção teórica entendida como produção social que prioriza a promoção da autonomia e a democratização das ações em saúde (Mendes, 1999; Paim, 2006).

A efetiva consolidação do SUS configura-se como possibilidade de mudança na forma com que os serviços e práticas de saúde se organizam, redirecionando enfoques e pautando-se em paradigmas voltados para a saúde enquanto expressão de cidadania. A reorganização da atenção à saúde no Brasil requer um acúmulo de forças capazes de reestruturar a produção de serviços e de conhecimento, submetendo-as ao bem comum. Sem perder de vista a complexidade dessa discussão, cabe ressaltar duas premissas integradas e inadiáveis: no âmbito da sociedade, faz necessário fortalecer o controle social sobre as ações governamentais e, no escopo da gestão pública, é necessário priorizar a oferta dos serviços a partir das necessidades de saúde da população. Cabe ressaltar que a articulação entre os níveis de atenção figura como ponto crítico a ser enfrentado, sob o risco de diminuição do acesso da população aos serviços de saúde.

A estruturação de redes de referência especializadas a partir da atenção básica é ingrediente importante para organização do sistema de saúde. Nesse artigo, questionam-se os limites e as possibilidades para se reestruturar os serviços de saúde no SUS a partir desse nível de atenção. Parte-se da hipótese de que a atenção básica - ação integrada de promoção, prevenção e recuperação da saúde da população -, embora possa 
se constituir em espaço político para produção de dinâmicas e tecnologias mais partilhadas de poder, tem tido pouca influência no reordenamento do mercado de bens e de serviços de saúde no Brasil. Isso compromete seu potencial para a inversão do modelo de atenção.

0 artigo tem como objetivos contextualizar a atenção básica na gestão da assistência à saúde no SUS e refletir teoricamente sobre a reorganização dos serviços a partir da Atenção Primária à Saúde (APS), considerando as dimensões econômicas das políticas sociais. Inicialmente, discorre-se sobre a forma com que a atenção à saúde se organiza no SUS, com destaque para as correlações de forças entre o público e o privado em saúde. Em seguida, teoriza-se sobre a articulação da atenção básica com média e alta complexidades. Entende-se a perspectiva de rede de serviços como ponto estratégico para a reorganização das ações de saúde, por influir na dimensão econômica do mercado de bens e de serviços. Buscam-se subsídios para localizar estrategicamente a relação entre atenção básica, média e alta complexidades como ponto nevrálgico para as renovações sugeridas, pela razão central de envolver disputas mais explícitas entre o mercado e o bem-comum, essencial para a conformação das políticas sociais. Nesse estudo, utiliza-se o termo Atenção Básica (AB) com o mesmo sentido de Atenção Primária à Saúde (APS).

\section{Metodologia}

Trata-se de estudo teórico e documental com abordagem qualitativa para desvelar e interpretar cenários (Minayo, 2005). Realiza-se revisão de literatura e análise de documentos oficiais de organismos nacionais e internacionais, que reafirmam a APS como prioridade para os sistemas de saúde das Américas. Foram sistematizados artigos científicos publicados em periódicos nas bibliotecas virtuais a partir de 1998, nos endereços eletrônicos www.bvs.br; www.scielo.br e http://www. periodicos.capes.gov.br, com uso das palavras-chaves atenção básica, atenção primária à saúde, SUS e gestão do sistema de saúde. Escolheu-se esse período por coincidir com o início da expansão do Programa Saúde da Família (PSF) no Brasil, que tem como propósito reorganizar os serviços de saúde a partir da atenção básica. Além disso, fez-se leitura de livros e autores relevantes para fundamentar a argumentação.
O estudo integra a pesquisa "Análise da relação dos serviços de média complexidade com a atenção básica à saúde do DF e entorno", financiada pela FAPDF, aprovada pelo Comitê de Ética em Pesquisa da Secretaria de Saúde do DF - CEP-DF o86/2005. Essa investigação avaliou a oferta e a demanda por serviços de média complexidade do DF, em sua relação com a atenção básica, por meio de survey e estudo de caso (Gottems e col., 2008).

\section{Gestão da Assistência à Saúde no SUS: atenção básica, média e alta complexidades}

A organização da assistência à saúde no SUS, por meio da rede de serviços de atenção básica $(\mathrm{AB})$, de média complexidade (MC) e de alta complexidade (AC), é realizada pela centralidade dos procedimentos médico-hospitalares sobre a promoção da saúde, preponderantemente. Tal característica é fruto de um modelo de atenção marcado pela hegemonia dos interesses da corporação médica, das indústrias e dos serviços privados de saúde. A tendência manifesta-se na compra indiscriminada de serviços do setor privado pelo público e na baixa qualidade da atenção básica do SUS, o que contribui para a ampliação do uso de planos privados pela classe média. Estudo oportuno sobre a oferta de serviços no Brasil (Santos e Gerschman, 2004), com foco nos arranjos institucionais, credores, pagadores e provedores, argumenta sobre a segmentação de clientelas segundo as lógicas de mercado. Acrescenta ainda que o poder de compra dos consumidores prepondera sobre os princípios do SUS, com diferenciações na qualidade e no tipo de serviço disponível.

No que se refere à concepção de atenção básica, recente portaria ministerial (Brasil, 2006) ratifica a visão clássica da APS enquanto primeiro nível de atenção, estratégia e filosofia para organizar os serviços de saúde (Starfield, 2002). Ela é descrita em Brasil (2006) como "um conjunto de ações de saúde, no âmbito individual e coletivo, que abrange a promoção e a proteção da saúde, a prevenção de agravos, o diagnóstico, o tratamento, a reabilitação e a manutenção da saúde”. Acrescenta-se o caráter democrático, participativo e integrado das ações, bem como os princípios que vêm norteando a organização dos serviços pela Estratégia Saúde da Família, como o trabalho em equipe, a territorialização, a corresponsabilidade sanitária, o foco nas necessidades da população e a Unidade Básica de 
Saúde (UBS) como contato preferencial dos usuários com o sistema de saúde.

Observam-se dualidades na política oficial, uma vez que a concepção ampliada do conceito se choca com a delimitação programática das ações, inicialmente proposta pela Norma Operacional de Assistência a Saúde (NOAS-SUS) 01/o2 (Brasil, 2002), e reeditadas no referido documento. Na conjuntura política e econômica em que a proposta de implantação do SUS se insere, convém considerar a atenção básica de forma ambígua, ou seja, ora como um conjunto de ações que pode viabilizar mudanças a partir dos seus princípios e em diálogo com os demais níveis, como advoga o discurso da Estratégia Saúde da Família (ESF), ora como programas de saúde pública, exequíveis por meio de atividades, atribuições e normas bem definidas.

A média e a alta complexidades inserem-se num cenário permeado por disputas mais acirradas entre o público e o privado. Numa primeira conceituação, a MC pode ser entendida como um conjunto de ações ambulatoriais e hospitalares, caracterizadas pela especialização médica, procedimentos diagnósticos e terapêuticos, adensamento tecnológico e oferta baseada na economia de escala. A economia de escala no setor saúde expressa-se na redução dos custos da produção, à medida que o volume ou a demanda por ações de saúde aumentam (Piola e Viana, 1995). Pautase, nessa lógica, a organização do SUS por níveis de complexidade, em que os serviços de atenção básica tendem a ser dispersos e próximos da população. Os de média e alta complexidades são regionalizados, localizados em emergências, ambulatórios de especialidades e internações hospitalares, visando à concentração do volume para redução de custos. Alie-se a isso o princípio da equidade no acesso, em que a primazia do direito à saúde se sobrepõe à eficiência econômica (Travassos, 1997). A alta complexidade é expressa como "um conjunto de procedimentos que, no contexto do SUS, envolve alta tecnologia e custo, objetivando propiciar à população acesso a serviços qualificados, integrando-o aos demais níveis de atenção (atenção básica e de média complexidade)" (Brasil, 2003). Vêse que esse conceito se define igualmente pelo fator econômico, pautando-se na regulação para otimizar a escassez da oferta.

As ações básicas de saúde, públicas, de menor custo e consumidas eminentemente pela população pobre, com baixo poder de vocalização e mobilização política, interferem muito pouco na relação entre mercado e trabalho, inerente à conformação das políticas sociais (Behring, 1988; Laurell, 2002). A média e a alta complexidades têm interface direta com o financiamento e manutenção do setor privado de serviços. O setor público financia parte das ações mais caras ofertadas pela rede de prestadores - a alta complexidade -, mantendo uma situação rentável para os hospitais privados, que corresponde a $61,8 \%$ dos estabelecimentos de saúde e mais de $60 \%$ dos leitos do Brasil (Brasil, 2004). Em linhas gerais, o SUS, forjado no seio do modo liberal-privatista de organizar a provisão de cuidados à saúde (Campos, 2007), continua comprando os procedimentos mais caros ofertados pela rede privada. Além disso, mantém baixa a concorrência entre os serviços públicos e privados de $\mathrm{AB}$ e $\mathrm{MC}$, possui clientela três vezes maior e com pouco poder de pressão. Para atender a esses usuários conta com uma oferta própria comparativamente menor, uma vez que apenas $42,6 \%$ dos estabelecimentos de saúde e 39,3\% dos leitos são públicos, e regula precariamente os prestadores de serviços (Matos e Pompeu, 2003).

\section{Intercessão do Político com o Econômico para Reorganização do SUS}

Nos últimos anos, em especial a partir da década de 1990 com a expansão do Programa Saúde da Família (PSF), discute-se que a mudança do modelo de atenção poderia ser feita a partir da atenção básica. Por modelo de atenção entende-se a forma com que a assistência à saúde é produzida e se estrutura nos serviços para atender às necessidades da população. Concebe-se pela articulação entre sujeitos (gestores, profissionais de saúde e usuários), tecnologias (conhecimentos aplicados) e recursos físicos (insumos, equipamentos, infraestrutura) utilizados no processo de trabalho para intervir sobre os problemas de saúde individuais e coletivos, contextualizados sócio-historicamente. Diz respeito ao modo de organizar as ações e os serviços de saúde, expressando-se no cotidiano das regras, normas e condicionantes macroeconômicos que conformam as políticas de saúde. 0 foco na autonomia dos sujeitos, na interdisciplinariedade e nas potencialidades partilhadas dos recursos locais, ampliando a noção de "clínica" para além do biológico, definiria a nova forma de agir em pauta, diferente da tradicional, em que a doença e a 
fragmentação dos processos de trabalho predominam sobre as necessidades dos usuários (Cunha, 2005; Paim, 2006).

Apesar do potencial de mudança que a atenção básica enseja, pelo menor aprisionamento das práticas, pela formação de vínculos com as famílias, pela capilaridade e poder de mobilização das redes sociais (Pires, 2004), parece não ser suficiente para mudar a lógica de produção das ações em saúde, considerando as disputas entre capital e trabalho mediadas pelo Estado na conformação das políticas sociais (Offe, 1991). A atenção básica precisaria de maior articulação entre as dimensões política e econômica da política de saúde para a reestruturação dos serviços no SUS. Para análise dessa premissa, verificam-se os principais argumentos que discutem a reorganização dos modelos assistenciais a partir da APS.

Desde a conferência de Alma-Ata, em 1978, que estabeleceu o lema "saúde para todos no ano 2000", a utopia de transformação de sistemas de saúde a partir das concepções e práticas ampliadas da APS inspirou movimentos de reforma sanitária em diversos países, inclusive no Brasil. Há consenso de que Alma-Ata influenciou, na década de 1980, reformas tanto em países de baixa renda, preponderantemente, como nos industrializados, em menor escala devido às melhores condições de saúde da população (Green e col., 2007). A conquista do SUS legal, fruto de movimento social envolvendo intelectuais, profissionais de saúde e setores médios da população, contemplou os ideais de justiça e democratização do sistema de saúde a partir da implantação de seus princípios (Fleury, 1997; Escorel, 1998). Recentemente, o não cumprimento da meta "saúde para todos no ano 200o" tem levado governos, profissionais de saúde, técnicos e intelectuais a refletirem sobre o tema.

A Organização Mundial da Saúde (OMS) e a Organização Pan-americana de Saúde (OPAS) ratificam a APS como eixo da reorganização dos sistemas de saúde, cujas diretrizes têm orientado as políticas de saúde de distintos governos. Recente publicação da OPAS reafirma a necessidade de renovação da atenção primária à saúde nas Américas, em consonância com as orientações da OMS (Macinko e col., 2007). Afirma-se, no referido ensaio, que existem evidências científicas para correlacionar atenção primária com a efetividade dos sistemas de saúde no mundo todo. Advoga-se pela adaptabilidade dos pressupostos aos distintos contextos sociais, políticos e econômicos dos países. Dentre os motivos para a renovação proposta, destacam-se: 1) a APS seria condição para alcance das metas do milênio, que são: acabar com a fome e a miséria; educação básica e de qualidade para todos; igualdade entre sexos e valorização da mulher; redução da mortalidade infantil; melhora da saúde das gestantes; combate à AIDS, à malária e a outras doenças; qualidade de vida e respeito ao meio ambiente (PNUD, 2007); 2) aproveitamento das lições aprendidas nos 25 anos decorridos desde AlmaAta; 3) surgimento de novos desafios epidemiológicos; 4) correção de alguns desvios nos enfoques divergentes da APS implementados em alguns países; 5) desenvolvimento de conhecimentos para tornar mais efetivas as práticas de APS; 6) reconhecimento de que a APS é uma ferramenta importante para reduzir desigualdades sociais e 7) a APS seria condição para enfrentar os determinantes sociais, alcançando altos níveis de saúde da população.

O documento da OPAS defende ainda compromissos, concepções, valores, princípios e elementos essenciais para um sistema de saúde baseado na APS, finalizando com as linhas estratégicas para a implantação da proposta. No campo das definições, considera que um sistema de saúde fundamentado na APS constitui uma estratégia de organização integral, pautada na saúde como direito universal, equitativo e solidário. Apresentam-se os princípios: a) intersetorialidade; b) participação; c) sustentabilidade; d) justiça social; e) responsabilidade e prestação de contas dos governos; f) orientação para a qualidade e g) respostas às necessidades da população. Enumeram-se os elementos essenciais: a) acesso e cobertura universais; b) atenção integral e continuada; c) ênfase na promoção e prevenção; d) atenção adequada; e) orientação familiar e comunitária; f) mecanismos de participação ativa; g) políticas e marco legal sólidos; h) organização e gestão ótimas; i) políticas e programas para promover equidade; j) contato preferencial aos serviços de saúde; k) recursos apropriados; l) recursos adequados e sustentáveis; e m) ações intersetoriais (Macinko e col., 2007).

Não obstante a relevância desses preceitos para o desenvolvimento de nações justas e éticas, percebe-se o tom ideológico das diretrizes da OMS/OPAS nem tanto pelo que afirma, mas pelo que não diz. Num dis- 
curso que cabe tanto à direita quanto à esquerda, as recomendações praticamente confirmam os equívocos presentes na declaração de Alma-Ata, ou seja, ratificar a ordem econômica mundial vigente e delegar unicamente para o espaço da política a tarefa de viabilizar políticas de saúde universais. Cabe acrescentar que o acesso universal e a atenção integral representam diretrizes que, em tese, são compatíveis tanto com o desenho socializante da tradição europeia dos sistemas nacionais quanto com a tradição norte-americana, liberal-privatista (Campos, 2007). Nem a declaração de 1978 nem a atual explicitam como subverter a atual mercantilização da saúde apenas pelo escopo da política, muito menos como alcançar a equidade em contextos em que prepondera o lucro das indústrias de bens e serviços de saúde concentradas nos países desenvolvidos.

Tendo em vista as atuais transformações sociais, políticas e econômicas do capitalismo, a discussão sobre o modelo assistencial é complexa, por envolver as repercussões ocorridas com o advento da globalização. A transnacionalização e a rápida circulação do capital financeiro, facilitadas pela conectividade das informações, modificaram as lógicas de produção, a acumulação capitalista e também o papel do Estado na regulação do mercado, que agora rompe as fronteiras nacionais e conforma um "império" com múltiplos focos de poder descentralizados (Hardt e Negri, 2002). Esse contexto macroeconômico repercute duramente nos países periféricos, como o Brasil, com influências sobre as políticas sociais (Pires e Demo, 2006).

A intocabilidade na ordem econômica mundial é explicitada em ambos os documentos, como se observa no trecho da declaração de Alma-Ata:

o desenvolvimento econômico e social baseado numa ordem econômica internacional é de importância fundamental para a mais plena realização da meta de saúde para todos e para a redução da lacuna entre o estado de saúde dos países em desenvolvimento e dos desenvolvidos. A promoção e proteção da saúde dos povos são essenciais para o contínuo desenvolvimento econômico e social e contribui para a melhor qualidade da vida e para a paz mundial (Conferência Internacional Sobre Cuidados Primários de Saúde, 1978).

Afirmar que a "ordem econômica internacional" é requisito para a realização da meta de "saúde para todos" revela certa incoerência. Seria possível garantir universalidade no direito à saúde apenas pelo viés do mercado, que se assenta na mais valia e na concentração de renda? Pelo que se tem visto, a primazia do lucro sobre o bem-comum, marca das economias liberais, tem alargado as desigualdades sociais no mundo todo (Santos, 2002). Na versão atual de Alma-Ata, o documento da OPAS é menos explícito na filiação ideológica. Uma leitura atenta, porém, desvela aspectos da primazia do mercado presentes nos compromissos assumidos pelas nações, na $18^{\circ}$ reunião da OPAS. Um deles, "o reconhecimento das funções decisivas tanto do indivíduo quanto da comunidade para estabelecer um sistema baseado na APS" (Macinko e col., 2007), embora seja requisito importante para o controle democrático da população sobre os poderes públicos, aponta certa tendência em individualizar e responsabilizar a sociedade privadamente por questões que são de ordem pública, requerendo a mediação essencial do Estado.

De todo, vislumbra-se uma situação ideal de saúde desconectada dos desafios globais que países periféricos da América, como o Brasil, têm de enfrentar. Algumas dessas questões centrais com que o SUS se depara são: a) importação da maioria dos equipamentos, medicamentos e insumos necessários à oferta de serviços de saúde, gerando dependência internacional; b) baixa competitividade do setor industrial em saúde; c) pouca capacidade produtiva em conhecimento e tecnologias, capaz de aumentar a autonomia e o desenvolvimento econômico; d) elevação da taxa de juros para atrair capital, comprometendo os investimentos internos; e) submissão das políticas sociais às determinações restritivas impostas por bancos, credores e FMI; f) contingenciamento orçamentário para pagamento da dívida externa e aumento dos fundos de reserva nacionais, gerando políticas públicas restritivas e focalizadas (Pires e Demo, 2006; Gadelha, 2003).

Para os dilemas da gestão do SUS - como o sub-financiamento das ações e serviços, a frágil regulação do setor privado, a incipiente política de desenvolvimento tecnológico e as iniquidades no acesso aos serviços -, as orientações dos organismos internacionais pouco contribuem para a redistribuição das desigualdades sociais. É pouco provável a coexistência de sistemas de saúde universais, equânimes e solidários com a concentração de renda e poder. Para ampliar as garantias sociais, é necessário haver intervenção no mercado 
de bens e serviços em favor do direito à saúde. Numa visão mais global, o desenvolvimento das capacidades produtivas do país, calcado no trabalho imaterial que se alimenta do conhecimento humano (Gorz, 2005; Lazzarato e Negri, 2001), é requisito central para aumentar a competitividade das nações periféricas no capitalismo global.

Observam-se nas justificativas para a "renovação da APS" certa limitação na aposta da relação entre saúde e desenvolvimento humano como condição determinante para o alcance das "metas do milênio". A afirmação de que a organização de sistemas de saúde fundados na atenção primária é suficiente para a redução de desigualdades sociais estruturais, como acabar com a fome, soa incompleta e dissociada do contexto sócio-histórico. Creditar ao setor saúde a responsabilidade de modificar questões complexas, como aumentar os níveis de educação do país ou reduzir as discriminações sociais, parece ter sido o principal equívoco da implantação do SUS (Conh, 2003). A escolha de um sistema "híbrido" entre o modo liberal e socializante de organizar o cuidado à saúde (Campos, 2007), em que o pagamento de prestadores privados de serviços hospitalares convive com a oferta de atenção básica eminentemente pública e gerida pelo Estado, tem limitado a consolidação do acesso universal e da atenção integral. A opção do Estado brasileiro de assumir a APS como estratégia para a "revolução social" (Campos, 2007), num contexto marcado por extremas desigualdades sociais condizentes com países periféricos, não tem sido suficiente para reordenar renda e poder. Nesses termos, é como se fosse possível fazer a revolução por dentro do Estado, algo controverso se o considerarmos, no final das contas, Estado capitalista, portanto comprometido também com o capital, embora palco para as conquistas sociais (Cocco, 2001).

Contrapondo-se aos argumentos apresentadas pelas correntes liberais, que concebem uma relação de causa e efeito entre saúde e desenvolvimento humano, como se ambos não fossem dialeticamente imbricados, Gadelha (2005) realiza consistente revisão teórica internacional. Ele cita três estudos curiosos que desmistificam a relação direta entre os indicadores de saúde e o crescimento econômico. 0 primeiro questiona a relação entre taxa de crescimento e mortalidade infantil, concluindo que a educação e o contexto institucional geral são mais centrais para as condições de saúde e o desempenho econômico das nações. O segundo não encontra evidência entre aumento da expectativa de vida e a renda per capita, indicando que, apesar de os países periféricos estarem com os indicadores de saúde um pouco melhores, a distância econômica acentua-se. 0 terceiro faz estimativas entre os PIB dos países, caso fossem eliminadas as adversidades de saúde. Conclui que, na situação hipotética de igualdade das condições de saúde, a diferença entre os 10\% mais ricos e os 10\% mais pobres cairia dos atuais 20,5 para 17,9, o que é irrisório.

O autor argumenta pela não incorporação da saúde como um fator genérico nas funções econômicas de produção. Parte da idéia de que é necessário repensá-la no contexto histórico-estrutural, na sua inserção global e na relação com a assimetria do progresso tecnológico. Localiza semelhanças entre a agenda liberal e a forma como os temas saúde e economia vêm sendo tratados no Brasil, ou seja, sujeição às falhas de mercado, tecnologias de baixo custo e complexidade, foco na atenção primária, municipalização e saúde como direito individual. À guisa de uma breve síntese, questiona: "um país e um povo pobre, dependente, desigual, sem acesso a conhecimento, com condições precárias de trabalho e sem capacidade de aprendizagem, mas que venha elevando sua expectativa de vida, é saudável?" (Gadelha, 2005, p. 332).

Se entendermos saúde na perspectiva históricoestrutural, resultado dos processos produtivos e das relações sociais, responde-se sonoramente não ao questionamento do autor. Para mudar as condições de pobreza, educação, trabalho, renda, poder e, portanto, saúde, numa nação democrática, é necessário mais do que sistemas de saúde fundamentados na APS. Ratifica-se a necessidade de se interferir nas lógicas desiguais de acumulação capitalista, buscando maior equidade no acesso às políticas sociais. Essa tarefa é complexa demais para se confinar ao idealismo dos valores, princípios e elementos da proposta de renovação da APS. Embora o espaço da política seja essencial para a pactuação das questões de interesse público, ele não ocorre destituído de poder e dos interesses privados, em especial no capitalismo. Para um embate de tal envergadura, há de se fortalecer o sentido do bem-comum e da cidadania na sociedade civil, situação ainda distante em países como o Brasil (Carvalho, 2004).

Cabe reafirmar que, para que a atenção primária à saúde possa conferir equidade e universalidade no 
acesso ao SUS, é preciso que ela seja suficientemente capaz de interferir no mercado de bens e de serviços de saúde. 0 potencial estratégico da relação entre os diferentes níveis de atenção do SUS reside na possibilidade de se agregar ao poder de vínculo e mobilização da comunidade, inerentes à APS, uma melhor regulação do sistema de saúde, em especial sobre a oferta de especialidades diagnósticas e terapêuticas, pertencentes à média e à alta complexidades. A atenção básica precisa de maior poder de resposta, por meio da incorporação de tecnologias e relação eficiente com as especialidades. Os valores, princípios e elementos da APS, destacados pela OPAS, seguem como utopia para o sistema de saúde brasileiro, tão duramente conquistado no bojo da democratização do país. Porém, cabe entendê-los na conjuntura neoliberal e globalizada, na perspectiva de vislumbrar estratégias capazes de ampliar a cidadania.

Argumenta-se que não é propriamente a atenção básica, mas a relação entre os níveis de atenção do SUS, o ponto estratégico para se operar as mudanças necessárias ao modelo de saúde vigente. A interface das UBS com urgências, serviços especializados e de diagnóstico confere maior adensamento tecnológico, resolubilidade e organização dos fluxos de usuários para continuidade da assistência. Uma vez mais bem articulada a $\mathrm{AB}$ com a MC, tende-se a impactar nas ações de alta complexidade do SUS, racionalizando a utilização dos serviços a partir das demandas da população. Por consequência, a relação do poder público com os prestadores privados de saúde terá de sofrer maior regulação por parte do Estado, interferindo no status quo de venda de serviços indiscriminada e pouco controlada (Matos e Pompeu, 2003).

Outro ponto importante refere-se ao impacto que um sistema público eficiente pode ter sobre a venda de planos privados de saúde. Se hoje essa comercialização ocorre, em muito, capitaneada pelas debilidades da atenção básica e da média complexidade do SUS, essa relação poderia mudar com uma oferta pública de maior qualidade. As melhores políticas sociais, no Brasil, são acessadas mais facilmente pelas classes média e alta, que detêm maior poder de vocalização de suas demandas (Demo, 2005). Não se advoga que ocorra um retrocesso na universalidade do SUS, ou que os ricos tomem o lugar dos pobres. Porém, a presença da classe média como usuária do sistema público de saúde tem se mostrado requisito importante para a conquista da cidadania. Como exemplo, cita-se o programa nacional de AIDS, referência mundial e palco importante dos movimentos organizados da sociedade civil, na sua relação com o Estado.

A luta iniciada pela Reforma Sanitária e demais atores sociais para a implantação do SUS precisa inserir as questões econômicas nos diversos espaços políticos de decisão para conquista do acesso à saúde universal. Para além das necessárias reivindicações pela ampliação do orçamento e regulamentação da Emenda Constitucional 29/200o (Santos, 2005), há de se incluir na pauta de negociação aspectos que se imbricam no mercado de bens e serviços de saúde, tais como: a) a relação com os prestadores privados de saúde; b) as políticas de investimentos para geração de conhecimentos e tecnologias para o setor; c) a submissão das emendas parlamentares à gestão do sistema de saúde; d) a regulação dos planos e seguros privados de saúde; e) a regulação da gestão do trabalho em saúde; f) a relação entre gastos públicos e resultados em saúde, dentre outros. Tratar a saúde como um bem público e capital social, portanto implicados nas relações de mercado, parece ser um caminho viável para a intercessão do político com o econômico rumo à sustentabilidade do SUS. Essa perspectiva transcende o discurso focalizado na APS como caminho para a implantação de sistemas de saúde universais.

\section{Considerações Finais}

A revisão teórica realizada corrobora a hipótese defendida neste estudo de que a atenção básica, embora possa se constituir em espaço político para produção de dinâmicas e tecnologias mais partilhadas de poder, tem tido pouca influência no reordenamento do mercado de bens e de serviços de saúde, comprometendo seu potencial para a inversão do modelo de atenção. No caso brasileiro, observa-se a limitação da atenção primária à saúde desencadear a reorganização dos serviços no SUS.

A atenção básica capaz de interferir na lógica da oferta a partir da demanda, promover maior acesso, equidade e racionalidade econômica ao SUS é aquela que melhor se articula com os serviços de urgência, especialidades e diagnose-terapia. Associe-se a isso uma população politicamente atuante, sabedora dos seus direitos e exercendo com firmeza o controle 
social sobre o governo na implementação de políticas sociais de qualidade. A inclusão da dimensão econômica na agenda da Reforma Sanitária aparece como componente central para o atual contexto globalizado e restritivo das garantias sociais. Eis um caminho estratégico para reorganização do sistema de saúde em pauta orientada pelos princípios e diretrizes que ratificam a saúde como direito inalienável, não sujeito às intempéries do mercado.

\section{Referências}

BEHRING, E. R. Política social no capitalismo tardio. São Paulo: Cortez, 1988.

BRASIL. Ministério da Saúde. Secretaria de Assistência à Saúde. Departamento de Descentralização da Gestão da Assistência. Regionalização da Assistência à Saúde: aprofundando a descentralização com equidade no acesso: Norma Operacional da Assistência à Saúde - NOAS-SUS 01/O2. 2. ed. Brasília, DF, 2002.

BRASIL. Ministério da Saúde. Média e alta complexidade. Brasília, DF, 2003. Disponível em: <http://portal.saude.gov.br/portal/sas/mac/area. cfm?id_area=821>. Acesso em: 23 nov. 2007.

BRASIL. Ministério da Saúde. Secretaria de Atenção à Saúde. Reforma do Sistema de Atenção Hospitalar Brasileiro. Brasília, DF, 2004. (Cadernos de Atenção Especializada).

BRASIL. Ministério da Saúde. Portaria GM/MS $n^{0} 648$, de 28 de março de 2006. Aprova a Política Nacional de Atenção Básica, estabelecendo a revisão de diretrizes e normas para a organização da Atenção Básica para o Programa Saúde da Família (PSF) e o Programa Agentes Comunitários de Saúde (PACS). Brasília, 2006. Disponível em:http://bvsms. saude.gov.br/bvs/publicacoes/prtGM648_20060328. pdf. Acesso em 15 jun. 2008.

CAMPOS, G. W. S. O SUS entre a tradição dos Sistemas Nacionais e o modo liberal-privado para organizar o cuidado à saúde. Ciência e Saúde Coletiva, Rio de Janeiro, v. 12, p. 1865-1874, 2007. Suplemento.

CARVALHO, J. M. Cidadania no Brasil: o longo caminho. Rio de Janeiro: Civilização Brasileira, 2004.
COCCO, G. Trabalho e cidadania: produção e direitos na era da globalização. São Paulo: Cortez, 2001.

CONFERÊNCIA INTERNACIONAL SOBRE CUIDADOS PRIMÁRIOS DE SAÚDE. Declaração de Alma-Ata. Alma-Ata, 1978. Disponível em: <http:// www.dhnet.org.br/direitos/sip/onu/saude/almaata. htm>. Acesso em: 23 nov. 2007.

$\mathrm{CONH}, \mathrm{A}$. Estado e sociedade e as reconfigurações do direito à saúde. Ciência e Saúde Coletiva, Rio de Janeiro, v. 8, n. 1, p. 9-13, 2003.

CUNHA, T. G. A construção da clínica ampliada na atenção básica. São Paulo: Hucitec, 2005.

DEMO, P. Dureza: pobreza política de mulheres pobres. Campinas: Autores Associados, 2005.

ESCOREL, S. Reviravolta na saúde: origem e articulação do movimento sanitário. Rio de Janeiro: Fiocruz, 1998.

FLEURY, S. A questão democrática na saúde. In: FLEURY, S. (Org.). Saúde e democracia: a luta do CEBES. São Paulo: Lemos, 1997. p. 25-41.

GADELHA, C. A. G. O complexo industrial da saúde e a necessidade de um enfoque dinâmico na economia da saúde. Ciência e Saúde Coletiva, Rio de Janeiro, v. 8, n. 2, p. 521-535, 2003.

GADELHA, C. A. G. Desenvolvimento e saúde: em busca de uma nova utopia. Saúde em Debate, Rio de Janeiro, v. 29, n. 71, p. 327-338, 2005.

GORZ, A. $O$ imaterial: conhecimento, valor e capital. São Paulo: AnnaBlume, 2005.

GOTTEMS, L. B. D. et al. Análise da relação dos serviços de média complexidade com a rede básica de saúde do DF e entorno: buscando prioridades para ampliar acessos e resolubilidades. Brasília, DF: Universidade de Brasília, 20o8. Relatório de pesquisa.

GREEN, A.; ROSS, D.; MIRZOEV, T. Primary health care and England: the coming of age of Alma Ata? Health Policy, Leeds (UK), v. 8o, n. 1, p. 11-31, 2007.

HARDT, M.; NEGRI, A. Império. Rio de Janeiro: Record, 2002.

IBGE - INSTITUTO BRASILEIRO DE GEOGRAFIA E ESTATÍSTICA. Pesquisa Nacional por Amostra de Domicílios PNAD. Brasília, DF, 2003. 
LAURELL, A. C. (Org.). Estado e políticas sociais no neoliberalismo. São Paulo: Cortez, 2002.

LAZZARATO, M.; NEGRI, A. Trabalho imaterial: formas de vida e produção subjetiva. Rio de janeiro: DP\&A, 2001.

MACINKO, J. et al. La renovación de la atención primaria de salud en las Américas. Revista Panamericana de Salud Publica, Washington, DC, v. 21, n. 2/3, p. 73-84, 2007.

MATOS, C. A.; POMPEU, J. C. Onde estão os contratos?: análise da relação entre os prestadores privados de serviços de saúde e o SUS. Ciência e Saúde Coletiva, Rio de Janeiro, v. 8, n. 2, p. 629-643, 2003.

MENDES, E. V. Uma agenda para a saúde. 2. ed. São Paulo: Hucitec, 1999.

MERHY, E. E. SUS e um de seus dilemas: mudar a gestão e a lógica do processo de trabalho em saúde: um ensaio sobre a macropolítica do trabalho vivo. In: FLEURY, S. (Org.). Saúde e democracia: a luta do CEBES. São Paulo: Lemos, 1997. p. 125-141.

MINAYO, M. C. S.; ASSIS, S. G.; SOUZA, E. R. Avaliação por triangulação de métodos: abordagem para programas sociais. Rio de Janeiro: Fiocruz, 2005 .

OFFE, C. Algumas contradições do Estado social moderno. In:____. Trabalho e sociedade: problemas estruturais e perspectivas para o futuro da sociedade do trabalho. Rio de Janeiro: Tempo Universitério, 1991. p. 113-131.

PAIM, J. S. Desafios para a saúde coletiva no século XXI. Salvador: EDUFBA, 2006.

PIOLA, S. F.; VIANA, S. M. (Org.). Economia da saúde: conceitos e contribuição para a gestão da saúde.

Brasília, DF: IPEA, 1995.
PIRES, D. Hegemonia médica na saúde e na enfermagem. São Paulo: Cortez, 1989.

PIRES, M. R. G. M. Politicidade do cuidado como referência emancipatória para a gestão de políticas de saúde: conhecer para cuidar melhor, cuidar para confrontar, cuidar para emancipar. 2004. Tese (Doutorado em Política Social) - Instituto de Ciências Humanas da Universidade de Brasília, Brasília, DF, 2004.

PIRES, M. R. G. M.; DEMO, P. Políticas de saúde e crise do Estado de bem-estar: repercussões e possibilidades para o SUS. Saúde e Sociedade. São Paulo, v. 15, n. 2, p. 56-71, 2006.

\section{PROGRAMA DAS NAÇÕES UNIDAS PARA O} DESENVOLVIMENTO. Declaração do milênio. Nova Iorque, 6-8 set. 200o. Disponível em: <http://www. pnud.org.br/odm/>. Acesso em: 15 jun. 2008.

SANTOS, B. S. Os processos de globalização. In: SANTOS, B. S. (Org.). Globalização e as Ciências Sociais. São Paulo: Cortez, 2002. p. 33-106.

SANTOS, N. R. Regulamentar a EC-29, avançar o modelo de gestão e realizar a universalidade com integralidade, eqüidade e participação. Saúde em Debate, Rio de Janeiro, v. 29, n. 71, p. 339-352, 2005.

SANTOS, M. A. B.; GERSCHMAN, S. As segmentações da oferta de serviços de saúde no Brasil: arranjos institucionais, credores, pagadores e provedores. Ciência e Saúde Coletiva, Rio de Janeiro, v. 9, n. 3, p. 795-806, 2004.

STARFIELD, B. Atenção primária: equilíbrio entre necessidades de saúde, serviços e tecnologia. Brasília, DF: Unesco: Ministério da Saúde, 2002.

TRAVASSOS, C. Eqüidade e o Sistema Único de Saúde: uma contribuição para debate. Cadernos de Saúde Pública, Rio de Janeiro, v. 13, n. 2, p. 325-330, 1997. 